\title{
Study of the Gas Kinematics in the Center of the Barred Spiral Galaxy NGC 4258.
}

\author{
S. N. Dodonov \\ Special Astrophysical Observatory, Russian Academy of Sciences, \\ Karachai-Cherkessian Republic, 357147, Russia
}

\begin{abstract}
Integral Field Spectrograph observations of southeast (SE) gas outflow in the center (11x13 arcsec) of NGC 4258 with spatial sampling 0.6 and 1.2 arcsec were made with the 6 -m Telescope. Reconstructed spatial and kinematic structure of the SE outflow from 20-30 pc to 200$220 \mathrm{pc}$ are presented.
\end{abstract}

\section{Introduction}

The Sbc galaxy NGC 4258 (M106) was included by Seyfert (1943) in his list of peculiar galaxies with UV excess and broad emission lines. After several years of discussions (Burbidge \& Burbidge 1962, Burbidge et al. 1963, Filippenko \& Sargent 1985, Courtes 1989, Pecontal 1990, Pecontal et al. 1992), it was established that two systems of broad and narrow lines are superimposed over the nuclear region and that the broad component lines are of order of $25 \AA$ wide while narrow line profiles are of order of $5 \AA$ wide. In recent papers, Cecil (1992) and Cecil et al. (1992) confirmed the double helical structure as braided jets, and a morphological study of NGC 4258 by Courtes et al. (1993) clarified the general structure of the galaxy. Recent VLBI observations (Miyoshi et al. 1995) give new data for understanding the nature of gas outflow.

\section{Observations and Data Reduction}

Observations were made with the Integral Field Spectrograph (IFS) (Afanasiev et al. 1990) on the 6-m Telescope in two observing runs: 25/26 May 1992 and 18/19 May 1994. Details are summarized in Table 1.

The IPCS was used in Time Resolved Imaging Mode (TRIM) in order to make high quality guiding of the observations possible. Data reduction was performed using the software developed for IFS in SAO RAS (Vlasiuk 1994). The spectrographic data after the preliminary reductions were used to create monochromatic maps of the central region of NGC 4258 in $\mathrm{H} \alpha,[\mathrm{NII}] \lambda 6548 / 6583$, [SII] $\lambda 6717 / 6731$, and [OIII] $\lambda 5007$. A galaxy map in the continuum $(\lambda 5300-$ 5400 ) was also obtained. For $\mathrm{H} \alpha$ and [NII], maps with a spatial sampling of 0.6 arcsec and 1.2 arcsec were obtained, with the former shown in Figure 1. The maps at 1.2 arcsec resolution for these filters and the others are shown in Figure 2. A Gaussian analysis of the emission lines was used to derive the velocity fields and velocity dispersion maps in [OIII], $\mathrm{H} \alpha,[\mathrm{NII}]$, and [SII]. The velocity field 
Table 1. Observational parameters

\begin{tabular}{|c|c|c|}
\hline Parameter & May 1992 & May 1994 \\
\hline Multi-lens array, squared lens & $9 \times 11$ & $9 \times 11$ \\
\hline Spatial sampling, arcsec/lens & 0.6 & 1.2 \\
\hline Field of view, arcsec & $4.5 \times 6.6$ & $11 \times 13$ \\
\hline Spectral resolution, $\AA$ pixel ${ }^{-1}$ & 2 & 1.6 \\
\hline Spectral range, $\AA$ & $6300-6900$ & $\begin{array}{l}4700-5300 \\
6200-6900\end{array}$ \\
\hline $\begin{array}{l}\text { Exposure } \\
\text { Seeing conditions, arcsec } \\
\text { Detector }\end{array}$ & $\begin{array}{l}2 \text { hours } \\
\approx 1 \\
\text { IPCS } 512 \times 512 \text { pix }\end{array}$ & $\begin{array}{l}40 \text { min \& } 1 \text { hour } \\
1 \\
\text { IPCS } 512 \times 512 \text { pix }\end{array}$ \\
\hline
\end{tabular}
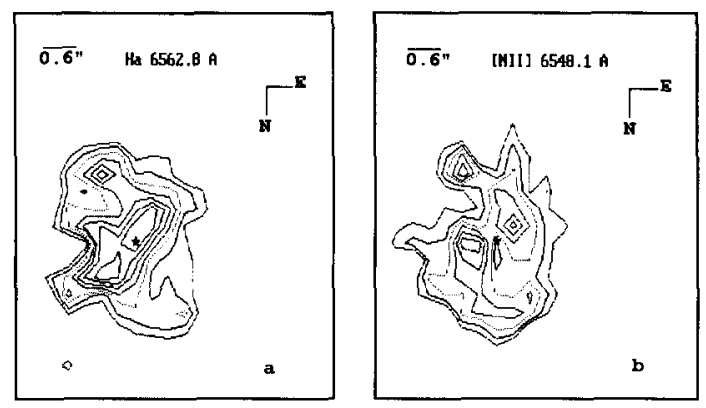

Figure 1. NGC 4258 monochromatic maps in emission lines (a) $\mathrm{H} \alpha$ and (b) [NII] $\lambda 6548 / 6583$. Spatial sampling is 0.6 arcsec. The center of the galaxy is marked by asterisk.

of the stellar disk was created using the cross-correlation of the Mgb $\lambda 5172 \AA$ region with a $\mathrm{K} 0$ template star spectrum. A systemic velocity of $462 \pm 5 \mathrm{~km} \mathrm{~s}^{-1}$ was estimated from these data for the center of the galaxy. In order to clarify the kinematic structure of the outflow we subtract the stellar disk velocity field from the velocity field of the ionized gas. Flux ratios [NII] $/ \mathrm{H} \alpha$ and $[\mathrm{SII}] / \mathrm{H} \alpha$ give us the possibility to study the gas conditions in the outflow region.

\section{Helical Structure of the SE Outflow}

The monochromatic maps show that the SE outflow is actually resolved in all observed emission lines. Structure of the outflow could be traced with our data from 20-30 pc to $200-220 \mathrm{pc}$. The rather complicated velocity field shows that the eastern edge of the outflow has systematically higher velocities than the western edge. This difference is $40-60 \mathrm{~km} \mathrm{~s}^{-1}$. We find that the width of the observed strands is $1.5-2 \operatorname{arcsec}(50-70 \mathrm{pc})$, the flux ratio [NII] $/ \mathrm{H} \alpha$ in the strands regions is near 1 , and that the strands step $1.5-2 \operatorname{arcsec}(50-70 \mathrm{pc})$ at the distance $2 \operatorname{arcsec}(70 \mathrm{pc})$, and $3-4 \operatorname{arcsec}(100-130 \mathrm{pc})$ at the distance 5-6 $\operatorname{arcsec}(160-200 \mathrm{pc})$ from the center of NGC 4258. These results suggest that we 
see helical motion and that a ballistic outflow model is less likely to be correct. Our attempt to restore from our data the spatial and kinematic structure of the outflow is shown in Figure 2f. No braiding is detected in our data. We could assume that the mean velocity of the outflow is of order $120-140 \mathrm{~km} \mathrm{~s}^{-1}$ in the center of the galaxy with a decrease to $80-100 \mathrm{~km} \mathrm{~s}^{-1}$ at the distance $4-5$ arcsec $(130-150 \mathrm{pc})$. In this case, the velocity gradient we observe between the eastern and western edges could be due to gas flow along the strands or a result of the rotation of the structure. Recent VLBI observations (Miyoshi et al. 1995) show that the spin axis of the molecular disk in the center of NGC 4258 is parallel to the $\mathrm{H} \alpha$ and radio synchrotron jets. The molecular disk and helical strands have the same sense of rotation. According to this we could assume that the genesis of the gas outflow is closely connected with the black hole in the center of NGC 4258 .
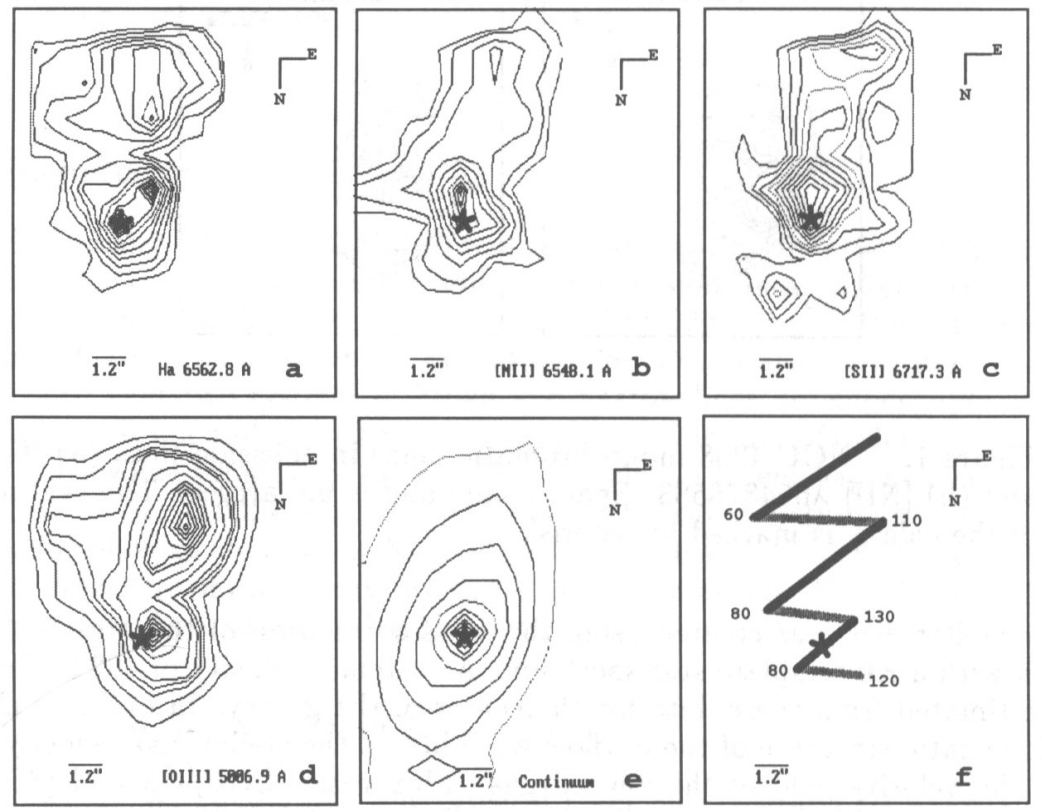

Figure 2. SE gas outflow region in the center of NGC 4258 : a) Monochromatic image in $\mathrm{H} \alpha \lambda 6562.8$; b) Monochromatic image in [NII] $\lambda 6548 / 6583$; c) Monochromatic image in [SII] $\lambda 6717 / 6731$; d) Monochromatic image in [OIII] $\lambda 5006.86$; e) Image in continuum $\lambda$ $5300-5500$; f) Spatial and kinematic structure of the SE outflow, restored from the observational data. Black colored strands represent the side of the outflow nearest to the observer, while the gray colored strands refer to the far side. Velocities on the edges of the outflow are marked in $\mathrm{km} \mathrm{s}^{-1}$; the regular velocity field of the stellar disk has been subtracted. 


\section{References}

Afanasiev, V. L., Courtes, G., Dodonov, S. N., \& Plana, H. 1994, Proc. of IAU Col. 149, 278-281.

Afanasiev, V. L., Vlasiuk, V. V., Dodonov, S. N., \& Sil'chenko, O. K. 1990, SAO Preprint 54

Burbidge, E. M. \& Burbidge, G. R. 1962, ApJ, 135, 694

Burbidge, E. M., Burbidge, G. R., \& Prendergast, K. H. 1963, ApJ, 137, 1022

Cecil, G., Wilson, A., \& Tully, R. B. 1992, ApJ, 390, 365

Courtes, G. 1989, IAU Coll. 120, 80

Courtes, G., Petit, H., Hua, C. T., Martin, P., Blecha, A., Huguenin, D., \& Golay, M. 1993, A\&A, 268, 419

Filippenko, A. V. \& Sargent, W. L. W. 1985, ApJS, 57, 503

Miyoshi, M., Moran, J., Herrnstein, J., Greenhill, L., Nakai, N., Diamond, P., \& Inoue, M. 1995, Nature, 373, 127

Pecontal, E. 1990, JAF, 37, 1

Seyfert, C. K. 1943, ApJ, 97, 28

Vlasiuk, V. V. 1994, in Tridimensional Optical Spectroscopic Methods in Astrophysics, G. Comte \& M. Marcelin, San Francisco: ASP, 308 